\title{
Automated Video Analysis of Handwashing Behavior as a Potential Marker of Cognitive Health in Older Adults
}

\author{
Ahmed Ashraf and Babak Taati \\ Version Post-Print/Accepted Manuscript \\ Publisher's Statement (C) 2016 IEEE. Personal use of this material is permitted. Permission \\ from IEEE must be obtained for all other uses, in any current or future \\ media, including reprinting/republishing this material for advertising or \\ promotional purposes, creating new collective works, for resale or \\ redistribution to servers or lists, or reuse of any copyrighted component \\ of this work in other works. (published version) Behavior as a Potential Marker of Cognitive Health in Older Adults. IEEE Journal of Biomedical and Health Informatics (IEEE JBHI), Vol. 20, No. 2, pp 682- 690, 2016. DOI: 10.1109/JBHI.2015.2413358 \\ Citation A. Ashraf and B. Taati. Automated Video Analysis of Handwashing
}

How to cite TSpace items

Always cite the published version, so the author(s) will receive recognition through services that track citation counts, e.g. Scopus. If you need to cite the page number of the TSpace version (original manuscript or accepted manuscript) because you cannot access the published version, then cite the TSpace version in addition to the published version using the permanent URI (handle) found on the record page. 


\title{
Automated monitoring of cognitive status by video analysis of handwashing behavior in older adults
}

\author{
Ahmed Ashraf ${ }^{1}$ and Babak Taati ${ }^{1,2}{ }^{*}$
}

\begin{abstract}
The identification of different stages of cognitive impairment can allow older adults to receive timely care and plan for the level of caregiving. People with existing diagnosis of cognitive impairment go through episodic phases of dementia requiring different levels of care at different times. Monitoring the cognitive status of existing patients is thus critical to deciding the level of care required by older adults. In this paper, we present a system to assess the cognitive status of older adults by monitoring a common activity of daily living (ADL), namely handwashing. Specifically, we extract features from handwashing trials of participants diagnosed with different levels of dementia ranging from cognitively intact to severe cognitive impairment as assessed by the mini mental state exam (MMSE). Based on videos of handwashing trials, we extract two classes of features: one characterizing the occupancy of different sink regions by the participant, and the other capturing the path tortuosity of the motion trajectory of participant's hands. We perform correlation analysis to assess univariate capacity of individual features to predict MMSE scores. To assess multivariate performance, we use machine learning methods to train models that predict the cognitive status (aware, mild, moderate, severe), as well as the MMSE scores. We present results demonstrating that features derived from hand washing behavior can be potential surrogate markers of a person's dementia, which can be instrumental in developing automated tools for continuously monitoring the cognitive status of older adults.
\end{abstract}

\section{Introduction}

Dementia, including Alzheimer's disease, is one of the biggest global public health challenges [1. As of 2013, over 35 million people worldwide live with

\footnotetext{
${ }^{* 1}$ Toronto Rehabilitation Institute, University Health Network, Toronto, Canada

${ }^{\dagger 2}$ Department of Computer Science, University of Toronto, Toronto, Canada
} 
the condition, and this number is projected to double by 2030 and more than triple to 135 million by 2050 [1. Some of the common forms of dementia include Alzheimer's disease, vascular dementia, dementia with Lewy bodies, and frontotemporal dementia [2, 3. Usual symptoms of dementia include memory loss, cognitive impairment, difficulty in communication, and mood changes among others [1, 2].

Primary care clinicians may not recognize cognitive impairment when using routine history and physical examination [4, 5] in as many as $76 \%$ of patients with dementia [6, 7, 8]. Most of these patients are not diagnosed until they are at moderate to severe stages of the disease [9].

Identification of different stages of cognitive impairment can potentially allow patients to receive timely care which could in turn lead to improved quality of life both for older adults and their caregivers who can better plan informal/formal care in advance. Persons with mild cognitive impairment usually continue to live at home, while people with moderate impairment start to need more significant caregiving. As a result, a timely identification of the cognitive status of older adults is of crucial value to allow them to plan the level of care. Persons with dementia typically exhibit mild symptoms in an inconsistent fashion when they are going through a phase of mild cognitive impairment [10. This makes the diagnosis even more challenging as patients can have normal outcomes in routine exams 4, 5. Dementia, along with its comorbidities, can adversely affect the patient's ability to carry out activities of daily living (ADL), but the symptoms of the disease may manifest with lesser severity and with inconsistent frequency during early stages of the disease.

Apart from affecting memory and language functions, Alzheimer's affects motor planning and hand movement [11, 12. Specifically, recent studies have noted a correlation between visuomotor impairment affecting eye-hand coordination in early-stage Alzheimer's disease [12. Moreover, symptoms of apraxia have been reported in patients with senile dementia of Alzheimer's type, which become apparent in patients with good language functions [11. Handwashing is a common ADL performed routinely requiring both motor planning and eye-hand coordination, and there has been some evidence that it is affected by cognitive impairment [13, 14. In this paper, we investigate if handwashing behavior can be a potential marker of cognitive disease.

A quantitative representation of the handwashing behavior may be formulated in multiple ways. To establish a proof-of-concept we first tested features derived from manually annotated handwashing trials for predicting cognitive status of the participants. We then explored if automated video analysis could be used for the same purpose. Specifically, we investigated features that encode the following aspects of the handwashing behavior: occupancy of different regions of the sink while completing the handwashing task, and the path tortuosity of the motion trajectory of the participant's hands. The latter class of features is inspired by a recent study which correlates the path tortuosity of walking patterns to the risk of falling in older adults [15]. As part of this study, we first showed that several of the above mentioned features have statistically significant correlations with the dementia level as assessed by the mini mental 
state exam (MMSE). We then trained machine learning models to predict the cognitive state categories based on the MMSE score (aware, mild, moderate, severe). We also trained regression models to predict the MMSE scores. Our results show that features encoding the above aspects of handwashing behavior can be potential predictors of cognitive impairment which can facilitate in developing tools for automated continuous monitoring of the cognitive status of older adults through a commonly executed ADL.

\section{Previous work}

Smart homes and home automation is increasingly catching traction that can assist in monitoring the health status of older adults [16, 17. Kaye et al have presented a system that monitors computer usage to assess cognitive impairment of the users based on their usage patterns [17. In another study, Dodge

et al monitored the walking patterns of older adults in their home setting and suggested that walking variability might be a predictor of mild cognitive impairment [16. Handwashing behavior has been studied previously to provide assistance to older adults while washing their hands. For instance, in a system presented by Hoey et al, hand motion is tracked using a camera while a person is washing hands, and assistance in the form of alerts is given to the user [13, 18. The prompting system in 18, tracks responsiveness to the prompts, and indirectly estimates the dementia level. Although promising, the study in [18 remained inconclusive whether the estimated levels of dementia correlated with the outcome of a standardized test such as the MMSE score. The focus of our current study is a standalone and passive monitoring system which can assess the cognitive health status of older adults that correlates with MMSE scores. Specifically, we investigate if the level of dementia could be predicted using hand washing behavior as parameterized by the following features: statistics of occupancy of different regions of the sink while handwashing, and the path tortuosity of motion trajectory of participant's hands.

\section{Materials and Methods}

\subsection{Study population}

We used a subset of the data analyzed in the study that investigated the effect of operator's familiarity with the faucet on difficulty of operation [14. All data was collected, stored, and analysed according to a protocol that was approved research ethics (IRB, University at Buffalo and REB, University of Toronto). A brief review of the data is as follows. The study population consisted of 27 older adults (22 female, 5 male), age range: $64-100$ years ( $82.4 \pm 9.5$ years). Each participant was administered the standardized MMSE exam [19] twice, once before the beginning, and once after the completion of the study. The average value of the two scores was taken, although the variation in the two scores was minimal (correlation between pre- and post-study MMSE scores was 0.98, $p<$ 
0.001). MMSE is a 30 point questionnaire test that is used to screen for dementia and cognitive impairment. The exam samples cognitive functions including arithmetic, memory, and orientation [19]. Low to very low MMSE scores have been reported to be correlated with the presence of dementia [20, 21, 22, Based on the MMSE score, the cognitive impairment of the test taker can be divided into four categories: severe ( $\leq 9$ points), moderate (10-18 points), mild (19-24 points), aware ( $\geq 25$ points).

\subsection{Hand washing trials}

Hand washing activity of the study participants was monitored in a designated washroom at a long-term care facility. The participants of the study were asked to wash their hands and videos of the sink area were recorded from an overhead view to enable post-trial analysis. Two researchers were present for all trials; one researcher acted as the caregiver, and the other researcher operated study-related equipment. For safety and study uniformity, the caregiver sat the participant in a wheelchair at the beginning of each trial. The caregiver positioned the participant at the sink and asked him or her to wash his or her hands. Each participant was asked to complete about 10 hand washing trials for five faucet types i.e. crosshead, dual lever, single lever, electronic, and plastic wand [14. In all, 1309 trials were conducted. For the purposes of this study, 741 trials that corresponded to commonly occurring faucet types i.e. crosshead, dual lever, and single lever, were included. Patient demographics of our study population with break down according to MMSE categories, age, and gender is given in Table 1 .

\begin{tabular}{|c|c|c|c|c|}
\hline & $\begin{array}{c}\text { Aware } \\
(M M S E \geq 25)\end{array}$ & $\begin{array}{c}\text { Mild } \\
(M M S E=19-24)\end{array}$ & $\begin{array}{c}\text { Moderate } \\
(M M S E=10-18)\end{array}$ & $\begin{array}{c}\text { Severe } \\
(M M S E \leq 9)\end{array}$ \\
\hline $\begin{array}{l}\text { Number of Participants } \\
\qquad(\mathrm{n}=27)\end{array}$ & $9(33 \%)$ & $3(11 \%)$ & $9(33 \%)$ & $6(22 \%)$ \\
\hline Female: $22(81 \%)$ & $5(56 \%)$ & $3(100 \%)$ & $9(100 \%)$ & $5(83 \%)$ \\
\hline Male: $5(19 \%)$ & $1(44 \%)$ & $0(0 \%)$ & $0(0 \%)$ & $1(17 \%)$ \\
\hline $\begin{array}{l}\text { Age range: } \\
64-100 \text { years }\end{array}$ & $64-86$ & $82-90$ & $68-95$ & $74-100$ \\
\hline $\begin{array}{l}\text { Age mean } \pm \text { Std: } \\
82.4 \pm 9.5 \text { years }\end{array}$ & $75.7 \pm 9.6$ & $85 \pm 4.4$ & $84 \pm 8.5$ & $86.3 \pm 10.4$ \\
\hline
\end{tabular}

Table 1: Demographics of study participants. 


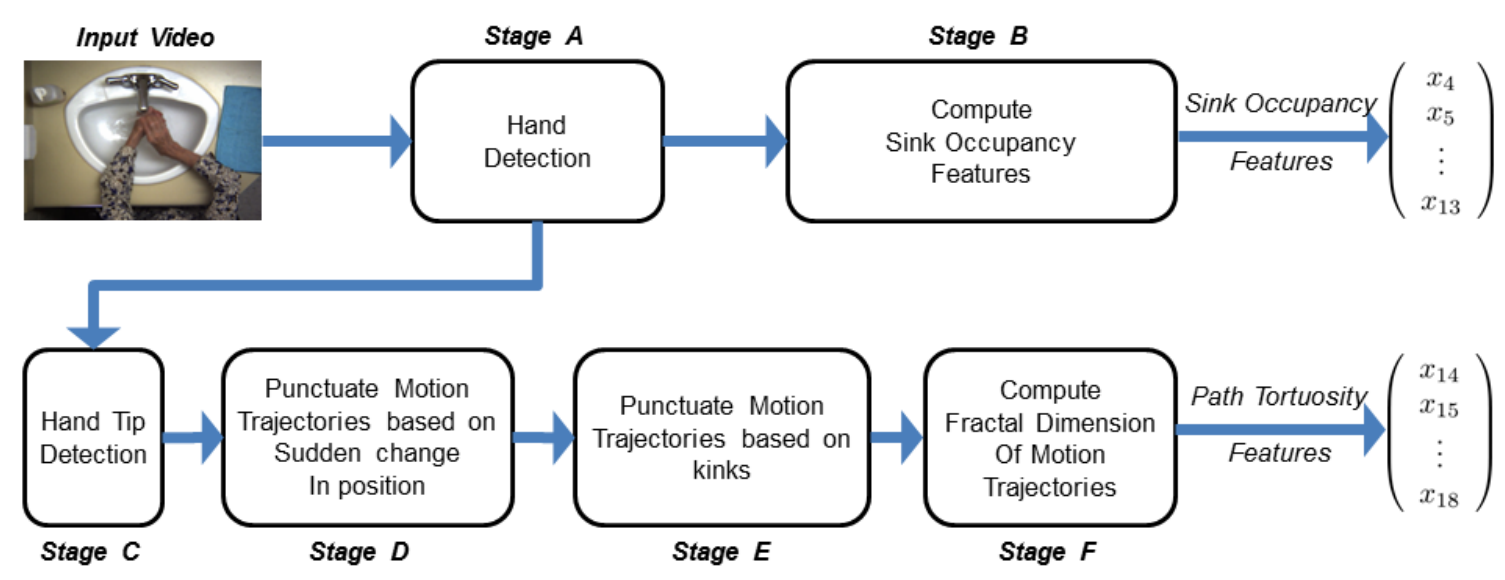

Figure 1: Block diagram of the feature extraction process.

\section{Proof-of-concept study}

To establish a proof-of-concept, the videos of handwashing trials were manually annotated as follows. A rater scored the number and types of errors in each handwashing trial [14. To validate data reliability, a second rater scored three randomly selected trials for each participant. Inter-rater agreement between the primary and secondary raters was examined using Cohen's kappa. Following features were computed from the manually annotated data: statistics of time taken to complete the handwashing task and the number and types of errors. A four class random forest classifier ([23]) was trained to predict the dementia level as aware, mild, moderate, or severe, which gave a classification accuracy of $61 \%$. Prompted by this moderate performance of the classifier using manually annotated videos we explored feature representations which could be extracted automatically, and could also characterize the handwashing behavior with more granularity. We present these features in the following section.

\section{$5 \quad$ Feature extraction for automated video analysis}

For automated video analysis of handwashing behavior we explored features characterizing the occupancy of different sink regions by the participant, and path tortuosity of the motion trajectory of participant's hands. Specifically, each handwashing trial was represented as a feature vector as follows. First, the faucet type was encoded as a categorical feature represented by a set of three binary variables. For instance, if the trial involved a crosshead faucet, 


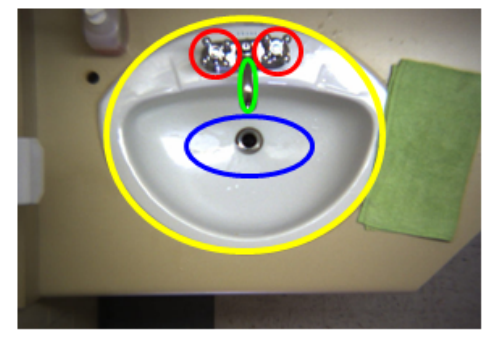

Crosshead faucet

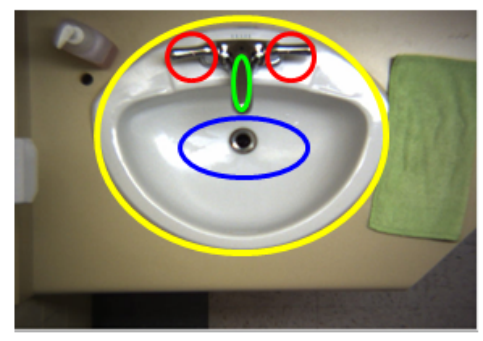

Dual lever faucet

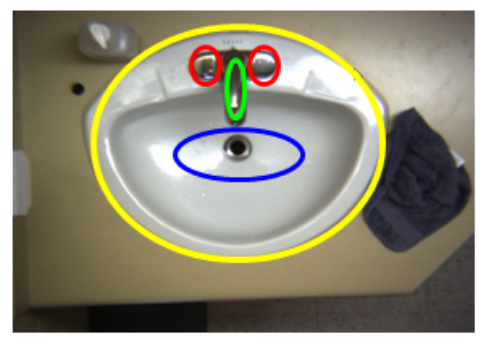

Single lever faucet

Figure 2: Definitions of different sink regions. Drain (Blue), Knob (red), Nozzle (green), Sink: subset of yellow region excluding all of the above, Sink (yellow).

it was encoded as $[1,0,0]$ etc. The three binary variables indicating the faucet type are shown as features 1-3 in Table 2. Thereafter, computer vision based features were extracted from each video. This feature extraction is a multistage process as depicted in the block diagram of Figure 1 Given an input video, the region corresponding to a participant's hands was identified by skin tone detection for every frame. The output of skin tone detection was post processed by a morphological opening step to eliminate small noisy specks [24. We then computed the following two types of features:

\subsection{Sink occupancy features}

Features included in this type capture the occupancy of various regions of the sink area as the participant performs the handwashing task. Specifically, we divided a video frame into sub-regions as illustrated in Figure 2, The 'Drain' region (shown in blue) constitutes a region centered around the sink drain. The 'Knob' region (shown in red) consists of two regions corresponding to the knobs of the cross-head faucet or the two levers of the dual-lever faucet. There are no knobs/levers in this region for a single-lever faucet, but we still analyze this region for a single lever faucet to potentially account for any confusion a participant might have. The 'Nozzle' region (shown in green) corresponds to the top of the faucet where one would find the lever for a singer lever faucet. ' $\overline{\text { Sink }}$ ' region is the area of the sink which does not correspond to any of the above regions. And finally, the 'Sink' region (shown in yellow) is the union of all the above regions.

The features that capture the statistics for occupancy of different regions are summarized in Table 2. For every region, the percentage area occupied by the hand per video frame was computed, and the average occupancy percentage for each region over the entire video was calculated (features 4-8 in Table 2). The time spent in different sub-regions of the sink was also estimated. To estimate the time, a sub-region was declared to be occupied if occupancy percentage exceeded a threshold. The number of frames for which a region was deemed occupied was then divided by the frame rate to get an estimate of the time 
spent in each region (features 9-13 in Table 2).

\begin{tabular}{|c|c|c|c|}
\hline$\#$ & Feature & Feature Description & $\begin{array}{l}\text { Correlation } \\
\text { with MMSE }\end{array}$ \\
\hline 1 & Crosshead lever & $\begin{array}{l}1 / 0 \text { to indicate if the faucet type is } \\
\text { Crosshead (c) or not }\end{array}$ & -0.013 \\
\hline 2 & Dual lever & $\begin{array}{l}1 / 0 \text { to indicate if the faucet type is Dual } \\
\text { lever (d) or not }\end{array}$ & -0.018 \\
\hline 3 & Single lever & $\begin{array}{l}1 / 0 \text { to indicate if the faucet type is Single } \\
\text { lever }(\mathrm{s}) \text { or not }\end{array}$ & 0.031 \\
\hline \multicolumn{4}{|c|}{ Sink Occupancy Features: (See Figure 2 for definition of sink regions) } \\
\hline 4 & Occ Drain & Percentage occupancy of the Drain region & $0.332^{*}$ \\
\hline 5 & $O c c_{K n o b}$ & Percentage occupancy of the Knob region & 0.005 \\
\hline 6 & $O c c_{N o z z l e}$ & Percentage occupancy of the Nozzle region & -0.058 \\
\hline 7 & $O c c_{\overline{\operatorname{Sink}}}$ & Percentage occupancy of the $\overline{\text { Sink }}$ region & $0.564^{*}$ \\
\hline 8 & $O c c_{\operatorname{Sink}}$ & Percentage occupancy of the Sink region & $0.554^{*}$ \\
\hline 9 & $t_{\text {Drain }}$ & Time spent in the Drain region & $-0.223^{*}$ \\
\hline 10 & $t_{K n o b}$ & Time spent in the Knob region & $-0.319 *$ \\
\hline 11 & $t_{N o z z l e}$ & Time spent in the Nozzle region & $-0.328^{*}$ \\
\hline 12 & $t_{\overline{\operatorname{Sink}}}$ & Time spent in the $\overline{\text { Sink }}$ region & 0.046 \\
\hline 13 & $t_{\text {Sink }}$ & Time spent in the Sink region & 0.007 \\
\hline \multicolumn{4}{|c|}{ Path Tortuosity Features: } \\
\hline 14 & $F D_{\text {Longest }}$ & $\begin{array}{l}\text { Fractal D of the longest trajectory (in } \\
\text { terms of duration) }\end{array}$ & $-0.410^{*}$ \\
\hline 15 & $F D_{\max }$ & Maximum fractal D & $-0.512^{*}$ \\
\hline 16 & $F D_{\min }$ & Minimum fractal D & -0.022 \\
\hline 17 & $F D_{\text {avg }}$ & Average fractal D & $-0.517^{*}$ \\
\hline 18 & $F D_{s t d}$ & Standard deviation of fractal D & $-0.423^{*}$ \\
\hline
\end{tabular}

Table 2: List of features extracted from each handwashing trial. The last column represents the Pearson's correlation of the individual feature with MMSE score. A (*) following the correlation represents the statistical significance of the correlation i.e, $p<0.05$

\subsection{Path tortuosity features}

Path tortuosity or variability in movement path, as assessed by the fractal dimension of the path, has been previously used to predict the risk of falls in older adults [15. Motivated by this study, we investigated if the path tortuosity of hand motion while washing could be a helpful feature for assessing cognitive health status.

For computing the path tortuosity, we first detected the tip of the hand 
based on the output of hand detection (stage A) as shown in the block diagram of Figure1. For tracking the tip of the hand, the top-most connected component (as assessed by the $y$-coordinate of its centroid) in the output of stage A was detected. The centroid of the top $10 \%$ pixels within this component was selected as the tip of the hand. It was observed that the skin detection algorithm was occasionally not perfect, and as a result did not allow to consistently detect the hand-tip. To avoid this problem, the motion trajectory was punctuated by points where the change in the position of the tip exceeded a threshold, effectively filtering out sudden motions. This step is carried out in stage D of the block diagram. A sample set of motion trajectories as detected by stage D are illustrated in the first row of Figure 3. As seen in the figure, there are a number of kinks in the trajectory, which can unnecessarily bias the fractal dimension of the trajectory toward higher values. As further processing, the motion trajectories were also punctuated by the presence of kinks as assessed by a curvature detection algorithm given in 25 . The outcome of this step is shown in the second and third rows of Figure 3. Very short motion segments in terms of duration (those that lasted less than 2 seconds) were discarded. Starting with the input video, the output of stage $\mathrm{E}$ is a collection of motion trajectories executed by the participant during the trial. The fractal dimension of each of these trajectories was computed using the box counting algorithm [26. Finally, the statistics of the fractal dimension were calculated for multiple motion trajectories executed during a trial as represented by features 14-18 in Table 2 ,

\subsection{Collapsed features}

The features as defined above are extracted from an individual video trial. In order to explore the variability in these features over multiple trials, for every participant all trials corresponding to a particular faucet type were collapsed to a single data point by computing the statistics (up to second order) of the features in Table 2. Effectively, all the trials for a particular participant were "collapsed" to three trials, each corresponding to a faucet type. For simplicity, from here on we would refer to the features computed at the level of individual videos as "uncollapsed" (Table 2), and the statistics of uncollapsed features

over multiple video trials as collapsed features. Features computed as part of the collapsed representation are summarized in Table 3.

\section{$6 \quad$ Predicting MMSE scores}

In this section we explore if the feature representations described above can predict the MMSE scores of participants. To analyze univariate performance of individual features, correlations of features with MMSE scores were evaluated, shown in the last columns of Tables 2 and 3 for uncollapsed and collapsed features respectively. Features with statistically significant correlations $(p<$ $0.05)$ are indicated by a '*' after their respective correlations. 


\section{0
0
0
0
0
$\frac{0}{0}$
5
5
$\frac{2}{5}$}

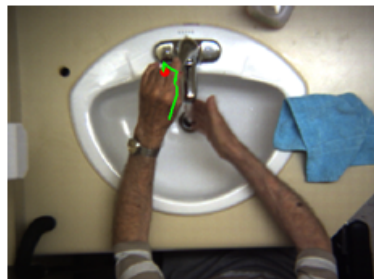

Motion Trajectory $1_{D}$

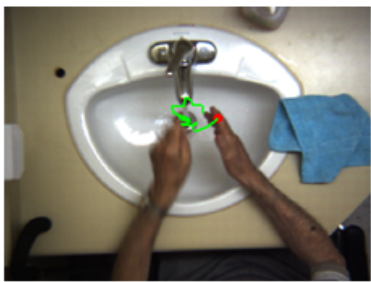

Motion Trajectory 2D

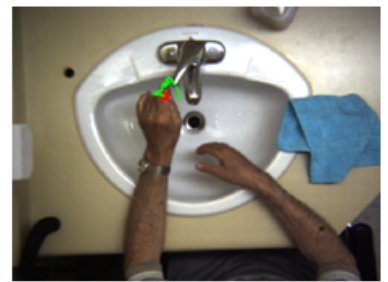

Motion Trajectory $3_{D}$

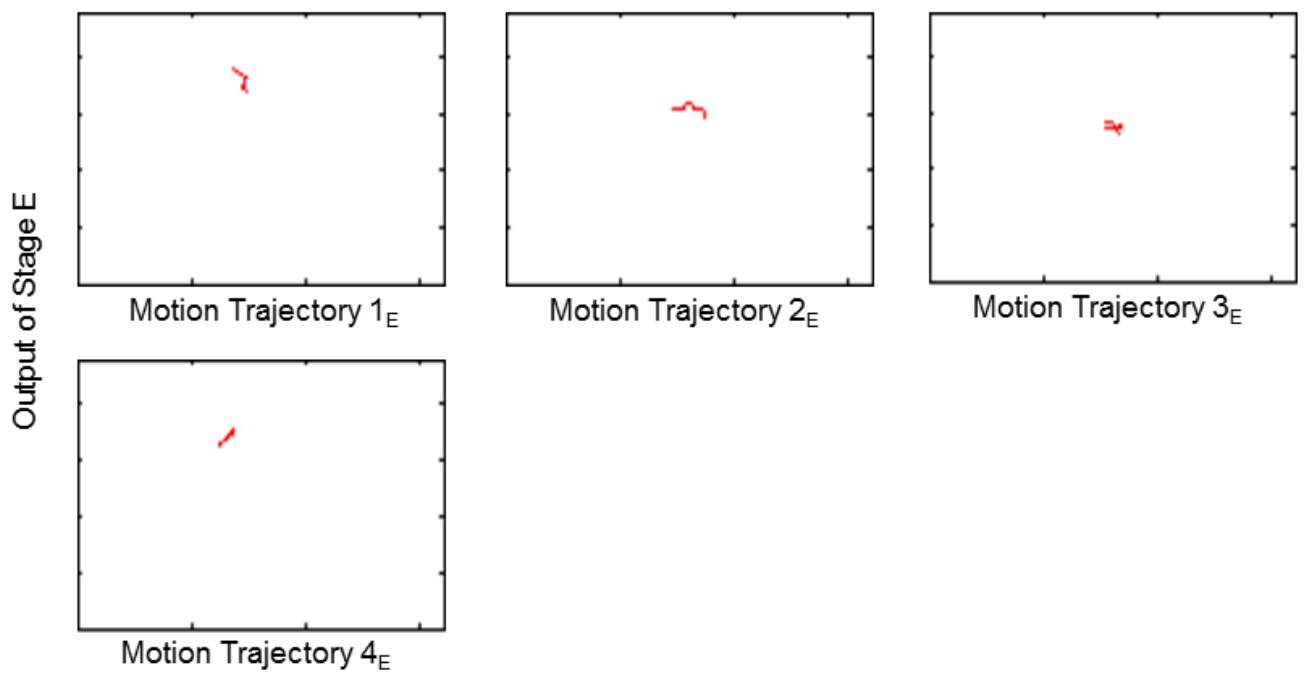

Figure 3: Detection of motion trajectories. Output of stage D: The first row shows the output of stage $\mathrm{D}$ where the motion trajectories (shown in green) are superimposed on the last video frame corresponding to that trajectory, and the red dot represents the hand-tip (Motion trajectory $1_{D}$ signifies the first motion trajectory in the output of step D). Output of stage E: Second and third rows show the output of stage $\mathrm{E}$ where the trajectories were punctuated by kinks, and very short motion segments were discarded. 


\begin{tabular}{|c|c|c|c|}
\hline$\#$ & Feature & Feature Description & $\begin{array}{l}\text { Correlation } \\
\text { with MMSE }\end{array}$ \\
\hline 1 & Crosshead lever & $\begin{array}{l}1 / 0 \text { to indicate if the faucet type is } \\
\text { Crosshead (c) or not }\end{array}$ & 0.000 \\
\hline 2 & Dual lever & $\begin{array}{l}1 / 0 \text { to indicate if the faucet type is Dual } \\
\text { lever (d) or not }\end{array}$ & 0.000 \\
\hline 3 & Single lever & $\begin{array}{l}1 / 0 \text { to indicate if the faucet type is Single } \\
\text { lever }(\mathrm{s}) \text { or not }\end{array}$ & 0.000 \\
\hline 4 & $\mu\left(O c c_{\text {Drain }}\right)$ & Mean \% occupancy of Drain region & $0.486^{*}$ \\
\hline 5 & $\sigma\left(O c c_{\text {Drain }}\right)$ & $\begin{array}{l}\text { Std deviation in \% occupancy of Drain re- } \\
\text { gion }\end{array}$ & -0.105 \\
\hline 6 & $\mu\left(O c c_{K n o b}\right)$ & Mean \% occupancy of Knob region & 0.010 \\
\hline 7 & $\sigma\left(O c c_{K n o b}\right)$ & $\begin{array}{l}\text { Std deviation in \% occupancy of Knob re- } \\
\text { gion }\end{array}$ & $-0.245^{*}$ \\
\hline 8 & $\mu\left(O c c_{N o z z l e}\right)$ & Mean \% occupancy of Nozzle region & -0.090 \\
\hline 9 & $\sigma\left(O c c_{N o z z l e}\right)$ & $\begin{array}{l}\text { Std deviation in \% occupancy of Nozzle re- } \\
\text { gion }\end{array}$ & -0.210 \\
\hline 10 & $\mu\left(O c c_{\overline{\text { Sink }}}\right)$ & Mean \% occupancy of $\overline{\operatorname{Sink}}$ region & $0.695^{*}$ \\
\hline 11 & $\sigma\left(O c c_{\overline{\operatorname{Sink}}}\right)$ & $\begin{array}{l}\text { Std deviation in \% occupancy of } \overline{\operatorname{Sink}} \text { re- } \\
\text { gion }\end{array}$ & 0.098 \\
\hline 12 & $\mu\left(O c c_{\text {Sink }}\right)$ & Mean \% occupancy of Sink region & $0.693^{*}$ \\
\hline 13 & $\sigma\left(O c c_{\text {Sink }}\right)$ & $\begin{array}{l}\text { Std deviation in \% occupancy of Sink re- } \\
\text { gion }\end{array}$ & 0.038 \\
\hline 14 & $\mu\left(t_{\text {Drain }}\right)$ & Mean time spent in Drain region & $-0.301^{*}$ \\
\hline 15 & $\sigma\left(t_{\text {Drain }}\right)$ & Std deviation of time spent in Drain region & $-0.595^{*}$ \\
\hline 16 & $\mu\left(t_{K n o b}\right)$ & Mean time spent in Knob region & $-0.472^{*}$ \\
\hline 17 & $\sigma\left(t_{K n o b}\right)$ & Std deviation of time spent in Knob region & $-0.507^{*}$ \\
\hline 18 & $\mu\left(t_{\text {Nozzle }}\right)$ & Mean time spent in Nozzle region & $-0.492^{*}$ \\
\hline 19 & $\sigma\left(t_{N o z z l e}\right)$ & Std deviation of time spent in Nozzle region & $-0.578^{*}$ \\
\hline 20 & $\mu\left(t_{\overline{\operatorname{Sink}}}\right)$ & Mean time spent in $\overline{\text { Sink }}$ region & 0.053 \\
\hline 21 & $\sigma\left(t_{\overline{\operatorname{Sink} k}}\right)$ & Std deviation of time spent in $\overline{\text { Sink }}$ region & $-0.345^{*}$ \\
\hline 22 & $\mu\left(t_{\operatorname{Sink} k}\right)$ & Mean time spent in Sink region & -0.003 \\
\hline 23 & $\sigma\left(t_{\operatorname{Sink}}\right)$ & Std deviation of time spent in Sink region & $-0.403^{*}$ \\
\hline 24 & $\mu(F D$ Longest $)$ & Mean fractal D of the longest trajectory & $-0.631^{*}$ \\
\hline 25 & $\sigma(F D$ Longest $)$ & $\begin{array}{l}\text { Std deviation in fractal } \mathrm{D} \text { of the longest } \\
\text { trajectory }\end{array}$ & $-0.469^{*}$ \\
\hline 26 & $\mu\left(F D_{\max }\right)$ & $\begin{array}{l}\text { Mean maximum fractal D over multiple tri- } \\
\text { als }\end{array}$ & $-0.689^{*}$ \\
\hline 27 & $\sigma\left(F D_{\max }\right)$ & $\begin{array}{l}\text { Std deviation in maximum fractal } \mathrm{D} \text { over } \\
\text { multiple trials }\end{array}$ & $-0.546^{*}$ \\
\hline 28 & $\mu\left(F D_{\min }\right)$ & $\begin{array}{l}\text { Mean minimum fractal D over multiple tri- } \\
\text { als }\end{array}$ & 0.011 \\
\hline 29 & $\sigma\left(F D_{\min }\right)$ & $\begin{array}{l}\text { Std deviation in minimum fractal } \mathrm{D} \text { over } \\
\text { multiple trials }\end{array}$ & $-0.538^{*}$ \\
\hline 30 & $\mu\left(F D_{a v g}\right)$ & Mean of average fractal D over multiple tri- & $-0.676^{*}$ \\
\hline 31 & $\sigma\left(F D_{a v g}\right)$ & $\begin{array}{l}\text { Sts dev in average fractal D over multiple } \\
\text { trials }\end{array}$ & $-0.522^{*}$ \\
\hline 32 & $\mu\left(F D_{s t d}\right)$ & $\begin{array}{l}\text { Mean of std dev in fractal D over multiple } \\
\text { trials }\end{array}$ & $-0.686^{*}$ \\
\hline 33 & $\sigma\left(F D_{s t d}\right)$ & $\begin{array}{l}\text { Std dev of std dev in fractal D over multiple } \\
\text { trials }\end{array}$ & $-0.267^{*}$ \\
\hline
\end{tabular}

Table 3: List of collapsed features. The last column represents the Pearson's correlation of the individual feature with MMSE score. A '*' following the correlation represents the statistical significance of the correlation i.e, $p<0.05$ 
To assess multivariate performance, a regression model was trained using leave-one-subject-out cross validation. Instead of ordinary least squares (OLS) linear regression, we used regularized regression based on least absolute shrinkage and selection operator (LASSO) 27. In LASSO regression, the sum of absolute values of the regression weights is constrained to be less than a threshold, which prevents regression weights from taking on very large values, thereby providing robustness against overfitting and inducing sparsity [27. Specifically, a regression model was trained using the training set corresponding to the current leave-one-out fold, and all trials of the left out subject were tested on this model to give a predicted MMSE score. Pearson's correlation coefficient was computed between the predicted and the actual MMSE score to assess the performance of the model. Regression models were trained for both the uncollapsed and collapsed feature representations.

The predicted MMSE scores using the regression model based on uncollapsed representation had a mild correlation $(R=0.663, p<0.001)$, while the model based on collapsed features had a moderate to strong correlation $(R=0.789, p<0.001)$ with the actual MMSE scores. Scatter plots between the predicted and ground truth MMSE scores for the model based on uncollapsed and collapsed features are shown in Figure 4

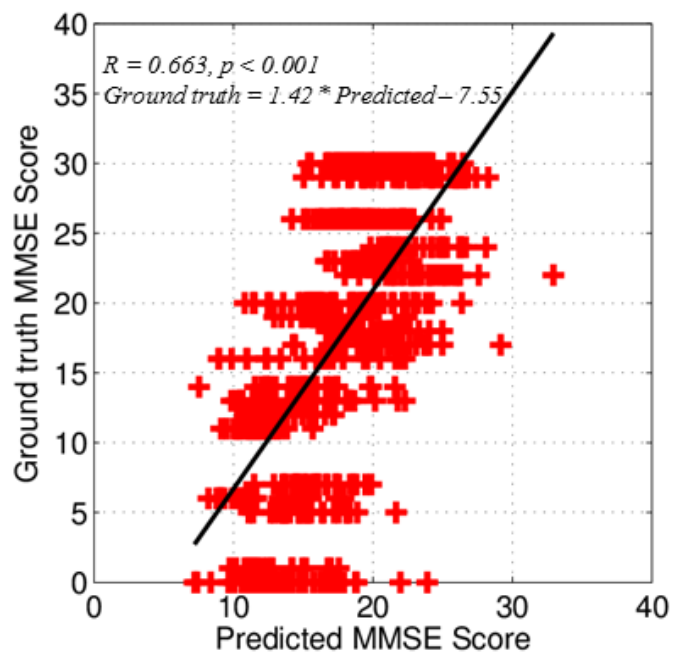

(a)

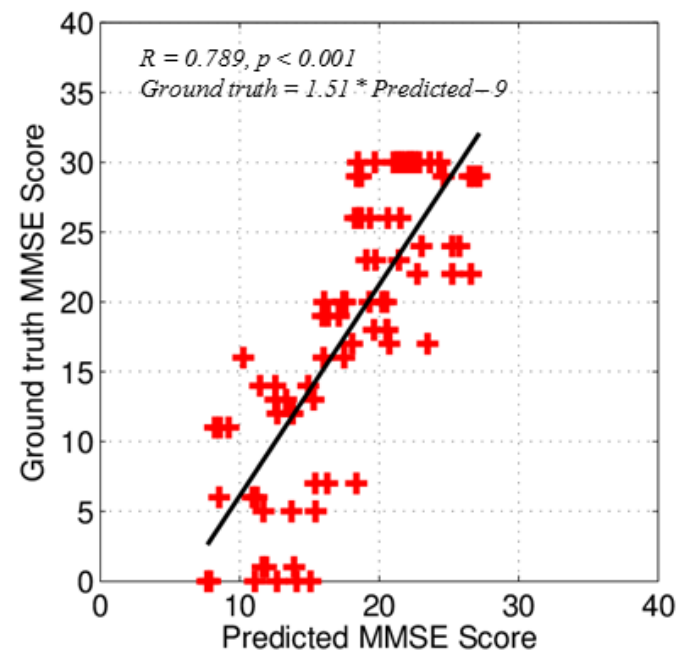

(b)

Figure 4: Scatter plot between the predicted MMSE and the ground truth MMSE scores based on leave-one-subject-out LASSO regression. (a) Model based on uncollapsed features, (b) Model based on collapsed features. 


\section{Predicting Dementia Categories}

While the performance of regression models was promising, in this section we investigate if the proposed features could also be used for predicting dementia categories as determined by MMSE scores. We followed a similar leave-onesubject-out cross validation scheme as mentioned in the previous section, but instead of regression, we trained random forest classifiers, 23, for predicting the dementia categories i.e. aware, mild, moderate, and severe. The leave-oneout classification results in terms of confusion matrices are given in Tables 4 and 5 for uncollapsed and collapsed feature representations respectively. The overall classification accuracy of the 4-class classifier with uncollapsed features was $52.1 \%$, while it was $70.4 \%$ for collapsed features.

To rank the features according to their relative importance, the random forest variable importance metric was employed [23]. Specifically, to measure the importance of a feature, the values of this feature are permuted among the training data, and the classification error is again computed on this permuted data. The importance of the feature is computed by averaging the difference in error before and after the permutation over all the trees in the forest. Since we used leave-one-out cross validation, the average rank of features was computed over all cross-validation folds. List of features arranged in ascending order of their rank is given in Table 6 .

\begin{tabular}{lcccccc}
\hline & \multicolumn{5}{c}{ Predicted } \\
& Aware & Mild & Moderate & Severe & $\%$ correct \\
\hline \multirow{2}{*}{ Aware } & 149 & 66 & 26 & 5 & $60 \%$ \\
Mild & 63 & 1 & 15 & 6 & $1.1 \%$ \\
Moderate & 60 & 12 & 146 & 36 & $58 \%$ \\
Severe & 5 & 3 & 58 & 90 & $57.7 \%$ \\
& & & & & $52.1 \%$ \\
\hline
\end{tabular}

Table 4: Classification results for predicting dementia categories based on leave-one-subject-out random forest classifier using uncollapsed features.

\section{Discussion}

In this study, we explored if computer vision based features encoding handwashing behavior of older adults can serve as surrogate predictors of their cognitive health. Our results show that the above mentioned features have moderate to strong correlation with the MMSE scores, and can also predict the dementia level as assessed by MMSE score based categories. The first observation that stems from our results is that collapsed feature representation outperforms the uncollapsed features both for regression $\left(R_{\text {collapsed }}=0.789\right.$ versus $R_{\text {uncollapsed }}=0.663$ ) and classification tasks (accuracy of $70.2 \%$ versus $52.1 \%$ ). 


\begin{tabular}{llccccc}
\hline & \multicolumn{5}{c}{ Predicted } \\
& Aware & Mild & Moderate & Severe & \% correct \\
\hline Aware & 21 & 5 & 1 & 0 & $77 \%$ \\
$\underset{\Xi}{\Xi}$ Mild & 7 & 0 & 2 & 0 & $0.0 \%$ \\
Moderate & 3 & 1 & 22 & 1 & $81.5 \%$ \\
Severe & 0 & 0 & 4 & 14 & $77 \%$ \\
& & & & & $70.4 \%$ \\
\hline
\end{tabular}

Table 5: Classification results for predicting dementia categories based on leave-one-subject-out random forest classifier using collapsed features.

This shows that statistics captured over multiple $(\sim 10)$ handwashing trials are more representative of cognitive health as compared to isolated single trials. This is in line with previous findings that about $76 \%$ of dementia patients go undiagnosed in routine primary care clinic visits [6, 7, 8, and highlights the importance of practical systems capable of monitoring cognitive health on a frequent basis.

In the confusion matrix of Table 5 , the classifier gives relatively high accuracies of $77 \%, 81.5 \%$ and $77 \%$ to detect aware, moderate, and severe categories respectively, pointing to the fact that it might be easier to distinguish more extreme categories. All mild cases were misclassified either as aware or moderate. There were only three participants (out of 27) with mild impairment in our data set. As a result, when videos of a participant with mild dementia were being tested, the leave-one-out model only had two participants to learn from. Poor performance in detecting mild cases can in part be because of the underrepresentation of participants with mild impairment in our data set and needs to be explored further.

Ranking of different features as shown in Table 6 needs further attention. Box plots of the variation of top 10 features are shown in Figure 5. Three main patterns emerge from these box plots. First, more cognitively intact participants tend to have higher percentage occupancy of different sink regions, as can be seen in the box plots of the features $\mu\left(O c c_{\text {Sink }}\right), \mu\left(O c c_{\overline{\text { Sink }}}\right)$ and $\mu\left(O c c_{\text {Drain }}\right)$. More interestingly, participants with moderate and severe level dementia have higher values of path-tortuosity (assessed by fractal dimension of the motion trajectories) as compared to participants who are cognitively aware or have mild dementia. This is evident in the box plots for the following features $\mu\left(F D_{\text {avg }}\right)$, $\mu\left(F D_{\max }\right)$, and $\mu\left(F D_{\text {Longest }}\right)$. This is consistent with a previous study in which the path tortuosity of walking patterns was indicative of cognitive health [15. Further, from the box plots of $\sigma\left(t_{\text {Drain }}\right)$ and $\sigma\left(t_{\text {Nozzle }}\right)$, it can be seen that participants with moderate and severe dementia have higher standard deviations in the time they spend in different sub-regions of the sink. This potentially points to the fact that inconsistent handwashing behavior in older adults might be representative of different levels of dementia. These findings are in line with 


\begin{tabular}{|c|c|c|}
\hline$\#$ & Feature & $\begin{array}{c}\text { Average } \\
\text { Feature Rank }\end{array}$ \\
\hline 1 & $\mu\left(O c c_{\operatorname{Sin} k}\right)$ & 1.72 \\
\hline 2 & $\mu\left(O c c_{\overline{\operatorname{Sink}}}\right)$ & 2.40 \\
\hline 3 & $\mu\left(F D_{\text {avg }}\right)$ & 2.40 \\
\hline 4 & $\sigma\left(t_{\text {Drain }}\right)$ & 3.76 \\
\hline 5 & $\mu\left(O c c_{\text {Drain }}\right)$ & 5.48 \\
\hline 6 & $\sigma\left(t_{K n o b}\right)$ & 6.80 \\
\hline 7 & $\mu\left(F D_{\max }\right)$ & 8.20 \\
\hline 8 & $\mu\left(F D_{s t d}\right)$ & 9.64 \\
\hline 9 & $\mu(F D$ Longest $)$ & 12.12 \\
\hline 10 & $\sigma\left(t_{\text {Nozzle }}\right)$ & 12.20 \\
\hline 11 & $\sigma\left(F D_{\min }\right)$ & 12.60 \\
\hline 12 & $\sigma\left(O c c_{\text {Drain }}\right)$ & 14.28 \\
\hline 13 & $\sigma\left(F D_{\max }\right)$ & 15.20 \\
\hline 14 & $\mu\left(F D_{\min }\right)$ & 16.48 \\
\hline 15 & $\sigma\left(F D_{a v g}\right)$ & 17.20 \\
\hline 16 & $\sigma\left(t_{\text {Sink }}\right)$ & 19.08 \\
\hline 17 & $\sigma(F D$ Longest $)$ & 19.72 \\
\hline 18 & $\sigma\left(O c c_{K n o b}\right)$ & 22.84 \\
\hline 19 & $\sigma\left(t_{\overline{\operatorname{Sink}}}\right)$ & 23.08 \\
\hline 20 & $\sigma\left(F D_{s t d}\right)$ & 23.76 \\
\hline 21 & $\mu\left(t_{\overline{\operatorname{Sink}}}\right)$ & 23.80 \\
\hline 22 & $\sigma\left(O c c_{\text {Sink }}\right)$ & 24.92 \\
\hline 23 & $\mu\left(t_{\text {Sink }}\right)$ & 25.60 \\
\hline 24 & $\mu\left(O c c_{N o z z l e}\right)$ & 25.68 \\
\hline 25 & $\mu\left(t_{K n o b}\right)$ & 26.44 \\
\hline 26 & $\mu\left(t_{\text {Nozzle }}\right)$ & 26.88 \\
\hline 27 & $\mu\left(t_{\text {Drain }}\right)$ & 27.08 \\
\hline 28 & $\sigma\left(O c c_{\overline{\text { Sink }}}\right)$ & 27.20 \\
\hline 29 & $\sigma\left(O c c_{\text {Nozzle }}\right)$ & 27.80 \\
\hline 30 & $\mu\left(O c c_{K n o b}\right)$ & 27.96 \\
\hline 31 & Crosshead lever & 28.40 \\
\hline 32 & Single lever & 30.04 \\
\hline 33 & Dual lever & 30.52 \\
\hline
\end{tabular}

Table 6: Average rank of features over all cross-validation loops 

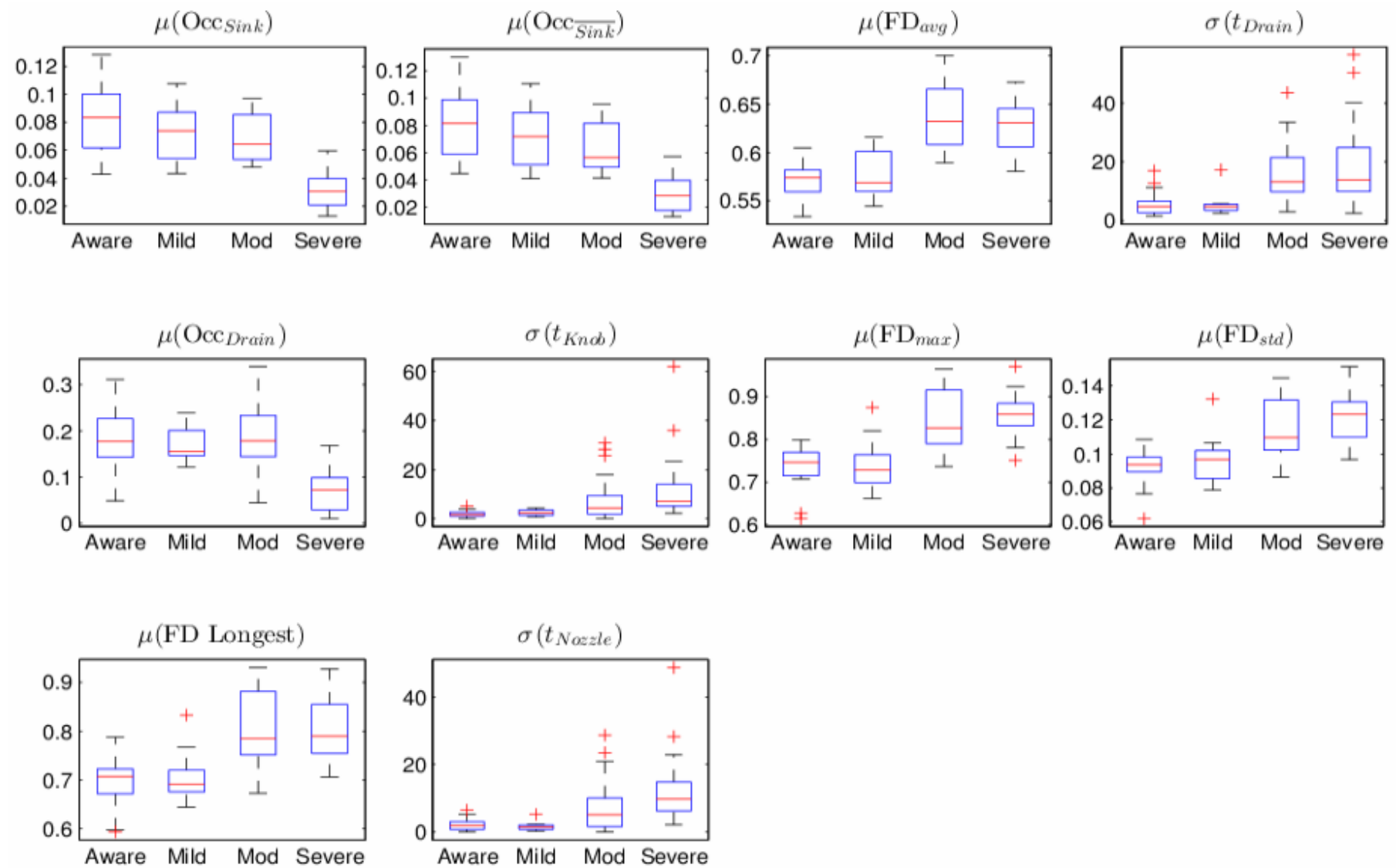

Figure 5: Box plots of highly ranked features showing the variation of these features across the four dementia categories. On each box, the central red mark is the median, the edges of the box are the 25 th and 75 th percentiles, the whiskers extend to the most extreme data points not considered outliers, and outliers are plotted individually as red pluses. 
[16, which reports variability in walking speed to be an indicator of cognitive impairment.

Some limitations of the study must be noted. Since the data used was initially collected for an earlier study, we did not have control over the study protocol. To assess the generalizability of results, future studies will incorporate cognitive tests other than MMSE scores as well as a test for apraxia, and will also control for comorbidities. In the current framework, a participant's hands are tracked by a skin-tone detection algorithm, which is not $100 \%$ accurate. In subsequent studies, 3D depth sensing could be employed for improved hand detection. Finally, in our current study, participants who fall in the mild dementia category (based on their MMSE score) are underrepresented. Future studies will work towards analyzing a larger population in which the number of participants from all categories are comparable.

\section{References}

[1] "Journey of caring: An analysis of long-term care for dementia," The World Alzheimer Report 2013, 2013. [Online]. Available: http: //www.alz.co.uk/research/WorldAlzheimerReport2013.pdf

[2] D. Shub and M. Kunik, "Psychiatric comorbidity in persons with dementia: Assessment and treatment strategies," Psychiatric Times, vol. 26, no. 4, April 2009.

[3] "Pathological correlates of late-onset dementia in a multicentre, community-based population in England and Wales. Neuropathology Group of the Medical Research Council Cognitive Function and Aging Study (MRC-CFAS)," Lancet, vol. 357, no. 9251, pp. 169-175, 2001.

[4] T. Holsinger, J. Deveau, M. Boustani, and J. W. Williams, "Does this patient have dementia?" JAMA, vol. 297, pp. 2391-2404, 2007.

[5] M. Ganguli, E. Rodriguez, B. Mulsant, S. Richards, R. Pandav, and J. V. Bilt, "Detection and management of cognitive impairment in primary care: The steel valley seniors survey," J Am Geriatr Soc., vol. 52, pp. 1668-1675, 2004.

[6] V. G. Valcour, K. H. Masaki, J. D. Curb, and P. L. Blanchette, "The detection of dementia in the primary care setting." Arch Intern Med., vol. 160, pp. 2964-2968, 2000.

[7] M. Olafsdottir, I. Skoog, and J. Marcusson, "Detection of dementia in primary care: the linkoping study." Dement Geriatr Cogn Disord., vol. 11, pp. 223-229, 2000.

[8] J. Chodosh, D. B. Petitti, M. Elliott, R. D. Hays, V. C. Crooks, and D. B. Reuben, "Physician recognition of cognitive impairment: evaluating the need for improvement." J Am Geriatr Soc., vol. 52, pp. 1051-1059, 2004. 
[9] A. J. Mitchell, "The clinical significance of subjective memory complaints in the diagnosis of mild cognitive impairment and dementia: a meta-analysis." Int J Geriatr Psychiatry., vol. 23, pp. 1191-1202, 2008.

[10] R. C. Petersen, G. E. Smith, S. C. Waring, R. J. Ivnik, E. G. Tangalos, and E. Kokmen, "Mild cognitive impairment: clinical characterization and outcome." Arch Neurol., vol. 56, no. 3, pp. 303-308, 1999.

[11] S. Rapcsak, S. Croswell, and A. Rubens, "Apraxia in alzheimer's disease," Neurology, vol. 39, no. 5, p. 664, May 1989.

[12] S. Verheij, D. Muilwijk, J. Pel, T. J. Cammen, F. U. Mattace-Raso, and J. Steen, "Visuomotor impairment in early-stage alzheimer's disease: changes in relative timing of eye and hand movements." J Alzheimers Dis., vol. 30, no. 1, pp. 131-43, 2012.

[13] A. Mihailidis, J. N. Boger, T. Craig, and J. Hoey, "The coach prompting system to assist older adults with dementia through handwashing: an efficacy study." BMC Geriatr., pp. 8-28, November 2008.

[14] J. N. Boger, T. Craig, and A. Mihailidis, "Examining the impact of familiarity on faucet usability for older adults with dementia," BMC Geriatr., vol. 13, no. 63, June 2013.

[15] W. D. Kearns, J. L. Fozard, M. Becker, J. M. Jasiewicz, J. D. Craighead, L. Holtsclaw, and C. Dion, "Path tortuosity in everyday movements of elderly persons increases fall prediction beyond knowledge of fall history, medication use, and standardized gait and balance assessments." J Am Med Dir Assoc., vol. 13, no. 7, p. 665, Sep 2012.

[16] H. Dodge, N. Mattek, D. Austin, T. Hayes, and J. Kaye, "In-home walking speeds and variability trajectories associated with mild cognitive impairment." Neurology, vol. 78, no. 24, pp. 1946-1952, June 2012.

[17] J. Kaye, N. Mattek, H. Dodge, I. Campbell, T. Hayes, D. Austin, W. H. K. Wild, H. Jimison, and M. Pavel, "Unobtrusive measurement of daily computer use to detect mild cognitive impairment." Alzheimers Dement., May 2013.

[18] J. Hoey, P. Poupar, A. von Bertoldi, T. Craig, C. Boutilier, and A. Mihailidis, "Automated handwashing assistance for persons with dementia using video and a partially observable markov decision process," Computer vision and image understanding, vol. 114, pp. 503-519, May 2010.

[19] D. Mungas, "In-office mental status testing: a practical guide," Geriatrics, vol. 46, no. 7, pp. 54-58, July 1991.

[20] S. Palmqvist, O. Hansson, L. Minthon, and E. Londos, "Practical suggestions on how to differentiate dementia with Lewy bodies from Alzheimer's disease with common cognitive tests," International journal of geriatric psychiatry, vol. 24, no. 12, pp. 1405-1412, December 2009. 
[21] A. L. Jefferson, S. A. Cosentino, S. K. Ball, B. Bogdanhoff, N. Leopold, E. Kaplan, and D. J. Libon, "Errors produced on the mini-mental state examination and neuropsychological test performance in alzheimer's disease, ischemic vascular dementia, and parkinson's disease," The Journal of neuropsychiatry and clinical neurosciences, vol. 14, no. 3, pp. 311-320, Summer 2002.

[22] T. A. Ala, L. F. Hughes, G. A. K. amd M. W. Ghobrial, and R. J. Elble, "The mini-mental state exam may help in the differentiation of dementia with lewy bodies and alzheimer's disease," International journal of geriatric psychiatry, vol. 17, no. 6, pp. 503-509, June 2002.

[23] L. Breiman, "Random forests," Machine Learning, vol. 45, no. 1, pp. 5-32, 2001.

[24] R. C. Gonzalez and R. E. Woods, Digital Image Processing (3rd Edition). Upper Saddle River, NJ, USA: Prentice-Hall, Inc., 2006.

[25] D. Chetverikov, "A simple and efficient algorithm for detection of high curvature points in planar curves," in Computer Analysis of Images and Patterns, ser. Lecture Notes in Computer Science, N. Petkov and M. Westenberg, Eds. Springer Berlin Heidelberg, 2003, vol. 2756, pp. 746-753.

[26] T. G. Smith, G. D. Lange, and W. B. Marks, "Fractal methods and results in cellular morphology dimensions, lacunarity and multifractals," Journal of Neuroscience Methods, vol. 69, no. 2, pp. 123-136, 1996.

[27] R. Tibshirani, "Regression shrinkage and selection via the lasso," Journal of the Royal Statistical Society, Series B, vol. 58, pp. 267-288, 1994. 